\title{
Estudio de la variabilidad genética del DRD4 Y del DAT 1 en individuos habitantes de la Ciudad de México
}

\author{
Gabriela Ariadna Martínez-Levy, ${ }^{1}$ Corina Benjet, ${ }^{2}$ Magdalena Briones-Velasco, ${ }^{1}$ \\ Amado Pérez-Molina, ${ }^{1}$ Andrés Nani, ${ }^{1}$ Carlos Sabás Cruz-Fuentes'
}

Artículo original

\section{SUMMARY}

Family, twin and adoption studies suggest that genetics plays an important role in the etiology of many psychiatric disorders. It has been proposed that the dopaminergic brain system could be affected in schizophrenia, substance abuse and attention deficit hyperactivity disorder. The most studied genes are two VNTR polymorphic systems; one located in the exon 3 of the dopamine D4 receptor (DRD4) gene, and the other in the $3^{\prime}$ untranslated region of the dopamine transporter (DAT1 or SCLA6A3) gene. It has been reported that allele frequencies of these polymorphisms varied between populations and this could affect the results in the association studies. Due to the previous findings, the objective of the present study was to determine the allele frequencies of DRD4 and DAT1 in an epidemiological sample of the adolescent population of México City. We found that the frequencies presented in our study were in between those reported for Caucasians and those reported for the American Indigenous population, this result are consistent with Euro-Indigenous inbreeding that has occurred in Mexico. Moreover, the results presented in the present study could explain the lack of consistency in the association analysis and make necessary to develop these investigations in our population.

Key words: DAT 1, DRD4, association, HWE.

\section{RESUMEN}

Existe evidencia fehaciente de la influencia genética en los trastornos psiquiátricos y se ha propuesto que el sistema dopáminergico cerebral puede ser uno de los afectados en diversos trastornos como la esquizofrenia, el abuso de sustancias y el trastorno por déficit de atención e hiperactividad. En este sentido, los sistemas genéticos más estudiados son 2 VNTRs; uno localizado en el exón 3 del gen del Receptor a dopamina D4 (DRD4) y el otro en la región $3^{`}$ no traducida del transportador a dopamina (DAT1 ○ SCL6A3). Se ha reportado que las frecuencias alélicas de estos polimorfismos difieren significativamente entre poblaciones y que esto puede afectar los resultados en los estudios de asociación. Debido a lo anterior, el objetivo del presente trabajo fue determinar las frecuencias alélicas del DRD4 y del DAT1 a partir de una muestra epidemiológica de la población adolescente de la Ciudad de México. Las frecuencias alélicas reportadas en el presente estudio son intermedias a las reportadas en caucásicos y poblaciones indígenas de América, lo que concuerda con la historia de mestizaje ocurrida en México. Estás diferencias pueden ayudar a explicar la falta de consistencia en diferentes estudios de asociación y hacen necesario realizarlos en población mexicana.

Palabras clave: DAT1, DRD4, asociación, HWE.

\section{ANTECEDENTES}

Múltiples estudios con familias, con gemelos y con sujetos dados en adopción han proporcionado evidencia fehaciente de la influencia genética en los trastornos psiquiátricos. ${ }^{1,2}$ Éstos presentan un patrón de herencia no mendeliana, compleja, con posible penetrancia incompleta y expresividad variable, que sugiere la acción conjunta de múltiples genes de efecto moderado o discreto con factores ambientales.,4

En la actualidad se conoce la estructura y localización de múltiples genes cuyos productos de expresión son fundamentales para el funcionamiento adecuado del Sistema
Nervioso Central. ${ }^{5,6}$ La variabilidad en estos genes puede afectar la expresión y/o estructura de la molécula transcrita y por lo tanto influir sobre los elementos emocionales, cognitivos y conductuales que son cardinales en la expresión de los síntomas o rasgos asociados a un tipo particular de trastorno psiquiátrico.

En particular, los genes y sus productos de expresión proteicos, que participan en el funcionamiento del sistema dopaminérgico cerebral, han sido considerados como interesantes candidatos relacionados con la manifestación de enfermedades mentales, como la esquizofrenia, el abuso de sustancias y el trastorno por déficit de atención e hiperacti-

Laboratorio de Genética, Subdirección de Investigaciones Clínicas, Instituto Nacional de Psiquiatría Ramón de la Fuente Muñiz.

Dirección de Investigaciones Epidemiológicas y Psicosociales, Instituto Nacional de Psiquiatría Ramón de la Fuente Muñiz.

Correspondencia: Dr. Carlos Cruz. Laboratorio de Genética, Subdirección de Investigaciones Clínicas, Instituto Nacional de Psiquiatría Ramón de la Fuente Muñiz. Calz. México-Xochimilco 101, San Lorenzo Huipulco, Tlalpan, 14370, México, DF. Fax. 5513-3722. E-mail: cruz@imp.edu.mx 
vidad (TDAH). Entre los genes más estudiados, relacionados con este sistema de neurotransmisión, se encuentran el receptor a dopamina D4 DRD4 y el transportador de dopamina (DAT1) $)^{2,5,6}$

El gen DRD4 codifica para una proteína transmembranal perteneciente al subgrupo de receptores dopaminérgicos tipo 2, y es miembro de la familia de las proteínas G inhibidoras unidas a receptores. Se han detectado altos niveles del RNA mensajero de esta proteína en la médula, la corteza prefrontal, el mesencéfalo, la amígdala y en los ganglios basales. ${ }^{7}$

Este gen se localiza en la región subtelomérica del brazo corto del cromosoma 11 y está constituido por cuatro exones. En su secuencia se han descrito diversos polimorfismos, entre los cuales destaca en particular un sistema polimórfico localizado en el tercer exón, que está constituido por secuencias repetidas en tandem (VNTR) de 48 pares de bases (pb). ${ }^{8}$ El número de repetidos en esta región varía de dos a 11, siendo la variedad de cuatro repetidos la más común. ${ }^{6,9}$

En particular, el alelo de 7R se ha asociado con diversas enfermedades mentales, como la esquizofrenia, el TDAH y el abuso de sustancias, así como con rasgos específicos de la personalidad como la "búsqueda de la novedad" ${ }^{6,7}$

Por otro lado, el gen DAT1 codifica para una proteína con 12 dominios transmembranales, regula la recaptura de dopamina del espacio sináptico y es el principal mecanismo propuesto para terminar la neurotransmisión dopaminérgica. ${ }^{10}$ Se ha descrito que los niveles de este transportador disminuyen con la edad y que la cantidad de este receptor se encuentra afectada en varios trastornos mentales, como la enfermedad de Parkinson, el síndrome de Gilles de la Tourette, la depresión mayor y el TDAH..$^{11}$ Asimismo, es uno de los blancos moleculares principales de drogas como la cocaína y las anfetaminas. ${ }^{10}$

El gen que codifica para el DAT1 se localiza en el cromosoma 5, en la región 15.3 del brazo corto, mide alrededor de $65 \mathrm{~kb}$ y se divide en 15 exones. Se han descrito varios polimorfismos en su secuencia; uno de los más estudiados es un VNTR de 40 pb localizado en la región 3` no traducida del gen. ${ }^{10-12} \mathrm{El}$ número de repetidos varía de 3 a 13, y se ha reportado que el alelo más común es el de 10 repeticiones. ${ }^{10,11}$ Este alelo común se ha asociado con diversos trastornos psiquiátricos, como abuso de sustancias y el TDAH. ${ }^{13}$

Es importante destacar que las frecuencias alélicas de ambos polimorfismos varían significativamente entre poblaciones $^{12-15}$ y que el alelo de riesgo reportado para algún trastorno mental puede variar de acuerdo con la población estudiada. ${ }^{16}$

Esto hace de sumo interés conocer las frecuencias alélicas de estos polimorfismos en la población mexicana para en un futuro poder realizar estudios de asociación con diferentes trastornos psiquiátricos, en específico el TDAH, que se ha asociado consistentemente con el alelo de $7 \mathrm{R}$ del gen DRD4 y el de 10R del gen DAT1. ${ }^{6,17-19}$

\section{MATERIAL Y MÉTODO}

Este estudio se realizó de acuerdo con los principios éticos descritos en la Declaración de Helsinki y fue aprobado por los comités Científico y de Ética del Instituto Nacional de Psiquiátrica Ramón de la Fuente Múñiz (INPRFM), localizado en la Ciudad de México.

\section{Sujetos}

Las muestras se obtuvieron de adolescentes que habitan en la zona metropolitana de la Ciudad de México, quienes participaron en un estudio epidemiológico realizado recientemente por el INPRFM, y que tuvo como objetivo estimar la prevalencia de las enfermedades mentales que se presentan de manera más frecuente en esta etapa del desarrollo. ${ }^{20}$

Tres mil cinco sujetos de entre 12 y 18 años fueron evaluados cara a cara por un entrevistador entrenado en el uso del CIDI-CAPI (Composite International Diagnostic InterviewComputer Assisted Personal Interview) en su versión adaptada para adolescentes. Esta entrevista generó un diagnóstico psiquiátrico, basado en los criterios del DSM-IV-R y el CIE10. Aquellos individuos que no cumplieron con los criterios para algún trastorno mental fueron escogidos como no casos. De este grupo se seleccionaron de manera aleatoria 116 individuos.

\section{Análisis molecular}

Se obtuvo una muestra de enjuague bucal de los individuos participantes y el ADN se extrajo con ayuda del Kit PureGene de Gentra para la purificación del ADN.

La amplificación del VNTR localizado en el exón 3 del gen DRD4 se llevó a cabo utilizando los primers reportados por Lichter ${ }^{21}$ y las condiciones descritas previamente por Aguirre..$^{22}$ Para el caso del VNTR localizado en la región $3^{`}$ no traducida del DAT1 se utilizaron los primers y el protocolo reportados previamente por Kang et al. (1999). ${ }^{12}$

La determinación del tipo de alelo se obtuvo de la electroforesis en geles de agarosa. El tamaño de los alelos se estableció comparando las bandas presentes en los geles con marcadores de peso molecular y/o con algunas muestras previamente secuenciadas. Dos investigadores distintos realizaron en forma ciega la lectura de los geles de agarosa y la mayoría de las muestras fueron amplificadas por lo menos dos veces con el objetivo de asegurar su correcta genotipificación. Los resultados dudosos no se incluyeron en el análisis.

\section{Análisis estadístico}

El equilibrio de Hardy-Weinberg (HWE) se analizó con la subrutina del programa HW incluido en el paquete estadístico LINKAGE. ${ }^{23}$ 
Cuadro 1. Frecuencias alélicas del polimorfismo tipo VNTR localizado en el exón 3 del gen DRD4 para diversas poblaciones humanas.

\begin{tabular}{|c|c|c|c|c|c|c|c|c|c|}
\hline & 2 & 3 & 4 & 5 & 6 & 7 & 8 & 9 & \\
\hline Caucásicos & 0.09 & 0.04 & 0.67 & 0.01 & 0.02 & 0.16 & 0.01 & 0.01 & $\begin{array}{l}\text { Chen et al., } 1999 \\
\text { Vyera et al., } 2003\end{array}$ \\
\hline Asiáticos & 0.15 & 0.01 & 0.79 & 0.03 & 0.02 & 0.01 & 0.00 & 0.00 & Chen et al., 1999 \\
\hline Africanos & 0.03 & 0.00 & 0.83 & 0.00 & 0.02 & 0.11 & 0.00 & 0.01 & Chen et al., 1999 \\
\hline Indígenas americanos & 0.03 & 0.00 & 0.29 & 0.01 & 0.03 & 0.60 & 0.02 & 0.00 & Vyera et al., 2003 \\
\hline Chilenos & 0.06 & 0.01 & 0.59 & 0.02 & 0.05 & 0.27 & 0.01 & 0.01 & Vyera et al., 2003 \\
\hline Este estudio & 0.03 & 0.01 & 0.58 & 0.02 & 0.01 & 0.35 & 0.00 & 0.00 & \\
\hline
\end{tabular}

\section{RESULTADOS}

De los 116 individuos que conformaron la muestra, sólo se obtuvieron los genotipos de 84 muestras para el DRD4 y 113 para el DAT1.

Las frecuencias genotípicas (datos no mostrados) de ambos polimorfismos estuvieron en equilibrio de HardyWeinberg (DRD4: $\chi^{2}=4.18$ g.1. $=15$ p=0.997; DAT1: $\chi^{2}=0.095$ g.l. $=1 \mathrm{p}=0.757)$.

Las frecuencias alélicas observadas en el presente estudio se muestran en los cuadros 1 (DRD4) y 2 (DAT1), así como su comparación con las frecuencias presentes en otras poblaciones. ${ }^{12-15}$

Con respecto al gen DRD4, se observa que al igual que en la mayoría de las poblaciones, a excepción hecha de los asiáticos, los alelos de 4 y 7 repetidos representan el 80-90\% de la diversidad alélica.

En particular, la frecuencia del alelo de $7 R$ del gen DRD4, que se ha asociado a diversos trastornos psiquiátricos, es mayor en nuestra población (35.1\%), que la reportada para caucásicos $(18 \%)$, pero mucho menor a la reportada para diversas poblaciones indígenas de América Latina (60\%) (cuadro 1).

Con respecto al gen DAT1, encontramos que en comparación con las poblaciones caucásicas existe un aumento del alelo de $10 \mathrm{R}$ en nuestra población ( $85.4 \%$ vs. $70 \%)$. Esto contrasta con lo reportado para poblaciones indígenas de Sudamérica, donde este alelo es prácticamente el único presente (95-100\%) (cuadro 2).

Cuadro 2. Frecuencias alélicas del polimorfismo tipo VNTR localizado en la región 3 `no traducida del DAT1 para diversas poblaciones humanas.

\begin{tabular}{lcccl}
\hline & 10R & 9R & Otros & \\
\hline Caucásicos & 0.70 & 0.29 & 0.01 & $\begin{array}{l}\text { Kang et al., 1999 } \\
\text { y Vyera et al., 2003 }\end{array}$ \\
Asiáticos & 0.91 & 0.06 & 0.03 & Kang et al., 1999 \\
Africanos & 0.37 & 0.28 & 0.35 & Kang et al., 1999 \\
Indígenas & 0.10 & 0.00 & 0.00 & $\begin{array}{l}\text { Kang et al., 1999 } \\
\text { s Vudamericanos }\end{array}$ \\
$\begin{array}{l}\text { Chilenos } \\
\text { Presente estudio }\end{array}$ & 0.74 & 0.23 & 0.03 & Vyera et al., 2003 2003 \\
\hline
\end{tabular}

El objetivo del presente estudio fue describir las frecuencias alélicas de dos sistemas polimórficos frecuentemente utilizados en los estudio de asociación y ligamiento genético con trastornos psiquiátricos.

Hasta nuestro conocimiento, éste es el primer reporte de las frecuencias alélicas del polimorfismo tipo VNTR de la región 3` no traducida del DAT1 en población mexicana. Por otro lado, los resultados obtenidos para el VNTR localizado en el exón 3 del gen DRD4 fueron similares a los publicados previamente por nuestro laboratorio, en un grupo independiente de individuos adultos, sanos mentalmente, que participaron como controles en un estudio de asociación con esquizofrenia. ${ }^{22}$

Es importante destacar que aunque el tamaño de la muestra es relativamente pequeño, es suficiente para estimar las frecuencias reales de una población. Además, debemos resaltar que las muestras provienen de un estudio epidemiológico, diseñado ex profeso, para obtener una muestra representativa tomando en consideración los distintos grupos de edad y nivel socioeconómico de la población adolescente de la Ciudad de México.

Las frecuencias genotípicas de ambos polimorfismos estuvieron en equilibrio de Hardy-Weinberg, lo que indica que es poco probable que las diferencias encontradas se deban a problemas de tipificación ${ }^{24,25}$ y refuerza la idea de que la población estudiada es sólo una, con lo que diminuye la posibilidad de problemas por estratificación poblacional. ${ }^{25}$

El hecho de que las frecuencias para ambos polimorfismos se encuentren intermedias entre las reportadas para las poblaciones caucásicas e indígena de América está en concordancia con la historia de mestizaje ocurrida en nuestro país desde la Colonia y muestra cómo está conformada genéticamente la población urbana de la Ciudad de México. Este comportamiento alélico es similar, por ejemplo, a lo que se ha reportado para estos mismos genes en la población urbana de Santiago de Chile. ${ }^{13}$

Como se mencionó anteriormente en población caucásica, los alelos de riesgo reportados para trastornos psiquiátricos como el TDAH son el de 7R del gen DRD4 y el de 10R del gen DAT1. 6,8,17,19 Es de destacarse que la frecuencia de estos alelos fue mayor en nuestra población. Esta caracterís- 
tica dificulta en todo caso el intentar replicar los resultados obtenidos en la población caucásica, ya que los tamaños de muestras requeridos para observar diferencias entre grupos de casos y controles se incrementa de manera importante. Sin embargo, es necesario recordar que existen reportes que indican que, dependiendo de la población estudiada, los alelos de riesgo pueden ser diferentes. En una población asiática se encontró que el alelo de 2R del gen DRD4 se encuentra asociado al TDAH, ${ }^{16}$ mientras que en las poblaciones caucásicas el alelo de riesgo es el de 7R.6,17-19

En conclusión, determinar las frecuencias alélicas representativas de una población de interés, para éstos y otros polimorfismos, es un requisito indispensable para poder entender el papel de los genes en los trastornos psiquiátricos.

Finalmente, los resultados del presente estudio proporcionan información sumamente relevante para generar un diseño experimental adecuado de los futuros estudios de asociación genética.

\section{AGRADECIMIENTOS}

A todo el personal del Laboratorio de Genética Psiquiátrica por su apoyo técnico en la realización del proyecto y en especial a Adriana Gómez Sánchez y a Graciela Mendoza de la Rosa por su apoyo en la extracción del ADN de las muestras. A Johanna McDermid por su apoyo en la revisión de la redacción del resumen en inglés. Al Posgrado en ciencias biológicas de la UNAM por el apoyo económico brindado a Gabriela Martínez para el desarrollo de su proyecto de maestría.

El presente trabajo fue realizado gracias al apoyo obtenido de CONACyT con el proyecto SEP-2004-COI-46594 y CB-2006-01-60678 y por el fondo de apoyo a la investigación 0196 del Instituto Nacional de Psiquiatría Ramón de la Fuente Muñiz.

\section{REFERENCIAS}

1. Asherson P, Curran S. Approaches to gene mapping in complex disorders and their application in child psychiatry and psychology. Br J Psychiatry 2001;179:122-128.

2. Kirley A, Hawi Z, Phil M, Daly G et al. Dopaminergic system genes in ADHD: Toward a biological hypothesis. Neurophsycopharmacology 2002;27(4):607-618.

3. Merikangas KR, Swedsen JD. Genetic epidemiology of psychiatric disorders. Epidemiol Rev 1997;19(1):144-155.

4. Caspi A, Moffitt TE. Gene-environment interaction in psychiatry: joining forces with neuroscience. Nat Rev Neurosci 2006;7:583-590.

5. Nicolini H, Sidenberg D, Camarena B, Guerra C et al. Evaluación de los genes del sistema dopaminérgico en esquizofrénicos mexicanos. Rev Invest Clin 1993;45:345-352.

6. Oak JN, Oldenhof J, Tol HHV. The dopamine D4 receptor: one decade of research. Eur J Pharmacol 2000;405:303-327.
7. D’Souza UM, Russ C, Tahir E, Mill J et al. Functional effects of a tandem duplication polymorphism in the 5 flanking region of the DRD4 gene. Biol Psychiatry 2004;56(9):691-697.

8. Van Tol HH, Bunzow JR, Guan HC, Sunahara RK et al. Cloning of the gene for a human dopamine $\mathrm{D} 4$ receptor with high affinity for the antipsychotic clozapine. Nature 1991;350(6319):610-614.

9. Wang E, Ding Y, Flodman P, Kidd JR et al. The Genetic Architecture of selection at the human dopamine receptor D4 (DRD4) gene locus. Am J Hum Gen 2004;73:931-944.

10. Fuke S, Suo S, Takahashi N, Koike $H$ et al. The VNTR polymorphism of the human dopamine transporter (DAT1) gene affects gene expresión. Pharmacogenomics J 2001;1(2):152-156.

11. Torres GE, Gainetdinov RR, Caron MG. Plasma membrana monoamine trasnporters: Structure, regulation and function. Nat Rev Neurosci 2003;4(1):13-25.

12. Kang AM, Palmatier MA, Kidd KK. Global variation of a $40 \mathrm{bp}$ VNTR in the 3 Untranslated region of the Dopamine Transporter gene (SLC6A3). Biol Psychiatry 1999;46:151-160.

13. Vieyra G, Moraga M, Henríquez H, Aboitiz F et al. Distribución de alelos de los genes DRD4 y DAT1 del sistema dopaminérgico en la población mixta de Santiago de Chile. Rev Med Chil 2003;131(2):135-143.

14. Chang FM, Kidd JR, Livak KJ, Pakstis AJ et al. The world-wide distribution of allele frequencies at the human dopamine D4 receptor locus. Hum Genet 1996;98(1):91-101.

15. Chen Ch, Burton M, Greenberger E, Dmitrieva J. Population Migration and the variation of Dopamine D4 receptor allele frequencies Around the Globe. Evol Hum Behav 1999;20:309-324.

16. Leung PWL, Lee CC, Hung SF, Ho TP et al. Dopamine receptor D4 (DRD4) Gene in Han Chinese children with attention deficit hyperactivity disorder (ADHD): Increased Prevalence of the 2-repeat allele. Am J Med Genet B Neuropsychiat Genet 2005;133B:54-56.

17. Comings DE, Chen TJ, Blum $K$, Mengucci JF et al. Neurogenetic interactions and aberrant behavioral co-morbidity of attention deficit hyperactivity disorder (ADHD): dispelling myths. Theor Biol Med Model 2005;2:50-65.

18. Todd RD, Huang H, Smalley SL, Nelson SF et al. Collaborative analysis of DRD4 and DAT genotypes in population-defined ADHD subtypes. J Child Psychol Psychiatry 2005;46(10):1067-1073.

19. Li D, Sham PC, Owen MJ, He L. Meta-analysis shows significant association between dopamine system genes and attention deficit hyperactivity disorder (ADHD). Hum Mol Genet 2006;15(14):2276-2284.

20. Benjet C, Borges G, Medina-Mora ME, Blanco J et al. La Encuesta Mexicana de Salud Mental Adolescente. En: Rodríguez J, Kohn R, AguilarGaxiola S (eds). La epidemiología de salud mental en América Latina y el Caribe. Washington D.C: Pan American Health Organization; 2009.

21. Lichter JB, Barr CL, Kenedy JL, Van Tol HH et al. A hypervariable segment in the human dopamine D4 receptor (DRD4) gene. Hum Mol Genet 1993;2(6):767-773.

22. Aguirre AJ, Apiquián R, Fresán A, Cruz-Fuentes C. Association analysis of exon III and exon I polymorphisms of the dopamine D4 receptor locus in Mexican psychotic patients. Psychiatry Res 2007;153(3):209-215.

23. Ott J. 1988-2001. Program HWE@. Utility Programs for Analysis of Genetic Linkage. Columbia University. http://linkage.rockefeller.edu/soft/linutil.

24. Hosking L, Lumsden S, Lewis K, Yeo A et al. Detection of genotyping errors by Ardí-Weinberg equilibrium test. E J Hum Genet 2004;12:395399.

25. Wigginton JE, Cluter DJ, Abecasis GR. A note on Exact Testo f HardeWeinber Equilibrium. Am J Hum Genet 2005,76:887-893.

Artículo sin conflicto de intereses 